\title{
ARTIGO ORIGINAL \\ Avaliação de variáveis relacionadas ao risco à hipertensão em indivíduos atendidos pelo Programa de Saúde da Família
}

Flaviany Custódio Faria*, Kátia Poles, D.Sc.**, Paula Midori Castelo, D.Sc. ${ }^{* *}$, Bruno Generoso Faria $^{* \star *}$, Eric Francelino Andrade, M.Sc. ${ }^{* \star * \star}$, Luciano José Pereira, D.Sc. ${ }^{* * * *}$

*Bacharel em Enfermagem, Centro Universitário de Lavras (UNILAVRAS), Lavras/MG, **Bacharel em Enfermagem, Docente no Departamento de Enfermagem, Universidade Federal de São João del Rei (UFSJ), Divinópolis/MG, ${ }^{* * * O d o n t o ́ l o g a, ~ D o c e n t e ~ n o ~ D e p a r t a m e n t o ~ d e ~}$ Ciências Biológicas do Instituto de Ciências Ambientais, Químicas e Farmacêuticas da Universidade Federal de São Paulo (UNIFESP), São Paulo/SP, ${ }^{* * * * M e ́ d i c o ~ V e t e r i n a ́ r i o, ~}$ Universidade Federal de Lavras, Lavras/MG, ${ }^{* * * *}$ Educador Físico, Universidade Federal de Lavras (UFLA), ${ }^{* * * * * * O d o n t o ́ l o g o, ~ D o c e n t e ~ n o ~ d e p a r t a m e n t o ~ d e ~ C i e ̂ n c i a s ~ d a ~ S a u ́ d e, ~}$ Universidade Federal de Lavras (UFLA)

Recebido em 18 de dezembro de 2014; aceito em 24 de julho de 2015.

Endereço para correspondência: Eric Francelino Andrade, Universidade Federal de Lavras, Campus Universitário, Caixa Postal 3037, 37200-000 Lavras MG, E-mail: ericfrancelinoandrade@gmail.com, Flaviany Custódio Faria, flafla_faria@hotmail.com, Kátia Poles,kpoles@usp.br, Paula Midori Castelo, pcastelo@yahoo.com, Bruno Generoso Faria, brunogenerosofaria@gmail.com, Luciano José Pereira, lucianojosepereira@dsa.ufla.br

\section{Resumo}

Objetivou-se com o presente estudo avaliar a inter-relação entre variáveis físicas, quadros de ansiedade/depressão e fatores socioeconômicos no acometimento de hipertensão em indivíduos atendidos em Programas de Saúde da Família. Uma entrevista foi realizada com 107 indivíduos, envolvendo questões relativas a dados pessoais e hábitos como tabagismo, etilismo, sedentarismo, idade, bem como fatores socioeconômicos e uso de medicamentos. Modelos de regressão logística (stepwise forward) foram empregados para avaliação dos fatores associados à variável dependente "hipertensão". Foi observado que o índice de massa corpórea, idade, consumo de álcool e níveis elevados de ansiedade aumentaram as chances de o indivíduo apresentar hipertensão, enquanto que o uso de medicamentos antidepressivos diminuiu ligeiramente este risco. Desta forma, conclui-se que hábitos de vida sedentários, associados ao tabagismo, ansiedade e consumo de álcool, correlacionam-se positivamente com a prevalência de hipertensão na amostra avaliada.

Palavras-chave: Saúde Pública, epidemiologia, modelos logísticos, promoção da saúde.

\section{Abstract \\ Evaluation of variables related to the risk of hypertension in individuals assisted by the Health Family Program}

The aim of this study was to evaluate the interrelation between physical variables, states of anxiety/depression and socioeconomic factors in the onset of hypertension in patients assisted in the Family Health Programs. An interview was conducted with 107 individuals, with questions related to personal data and habits like smoking, alcohol consumption, sedentary lifestyle, age, socioeconomic factors and medication consumption. Logistic regression models (stepwise forward) were used to assess factors associated with the dependent variable "hypertension". It was observed that the body mass index, age, alcohol consumption and high levels of anxiety have increased the chances of the individual presenting hypertension, while the use of antidepressant drugs decreased slightly this risk. Thus, we conclude that sedentary lifestyle, associated with smoking, anxiety and alcohol consumption, correlate positively with the prevalence of hypertension in the study sample.

Key-words: Public Health, epidemiology, logistic models, health promotion. 


\section{Resumen \\ Evaluación de las variables relacionadas con el riesgo de hipertensión en las personas atendidas por el Programa Salud de la Familia}

El objetivo de este estudio fue evaluar la interrelación entre variables físicas, estados de ansiedad/depresión y los factores socioeconómicos en la aparición de la hipertensión en pacientes atendidos en los Programas de Salud de la Familia. Se realizó una entrevista con 107 individuos, con preguntas relacionadas con los datos personales y hábitos como el tabaquismo, consumo de alcohol, el sedentarismo, la edad y los factores socioeconómicos y el uso de medicamentos. Se utilizaron modelos de regresión logística (stepwise forward) para evaluar los factores asociados con lavariable "hipertensión" dependiente. Se observó que el índice de masa corporal, la edad, el consumo de alcohol y altos niveles de ansiedad han aumentado las posibilidades de la hipertensión en los individuos, mientras que el uso de los fármacos antidepresivos disminuyó ligeramente este riesgo. Por lo tanto, se concluye que el estilo de vida sedentario, asociado con el tabaquismo, la ansiedad y el consumo de alcohol, se correlaciona positivamente con la prevalencia de la hipertensión en la muestra del estudio.

Palabras-clave: Salud Pública, epidemiología, modelos logísticos, promoción de la salud.

Introdução

A hipertensão arterial é um dos maiores problemas de saúde pública no Brasil por sua magnitude, risco e dificuldades no seu controle. É definida como uma doença multifatorial, sendo capaz de gerar respostas comportamentais das mais variadas ao portador. Estima-se que sua prevalência seja da ordem de $20 \%$ a $45 \%$ em algumas cidades brasileiras [1,2].

Em relação aos fatores de risco como idade, hereditariedade, raça e sexo, pouco pode ser feito, pois a hipertensão tem um forte componente familiar genético. Adicionalmente, idosos são mais acometidos que indivíduos jovens $[3,4]$. Porém, outros fatores são passíveis de alteração: estilo e qualidade de vida, hábitos como tabagismo e etilismo [5], alimentação inadequada [6], sedentarismo [7], estresse físico e psicológico [8], aumento dos triglicerídeos e colesterol séricos, obesidade e diabetes mellitus $[9,10]$.

A preocupação mundial em ampliar e aperfeiçoar os métodos para o tratamento da hipertensão arterial tem sido constante. Porém, faz-se necessário diagnosticar e classificar este distúrbio para determinados grupos de assistência em Saúde Pública, conforme sua etiologia e principais fatores de risco para verificar o melhor e mais adequado tratamento a ser instituído para todos os indivíduos que apresentarem a doença naquela localidade [11]. Frente aos dados evidenciados, os profissionais que atuam nos Programas de Saúde da Família (PSF) poderão propor medidas específicas, tais como estabelecimento de protocolos, educação continuada, medidas educativas junto aos grupos operativos em saúde, entre outras, beneficiando a população de cobertura da unidade.

Nesse contexto, os enfermeiros que atuam junto aos hipertensos no PSF têm papel importante no sentido de caracterizar a problemática dos hipertensos atendidos, levando em consideração todos os aspectos relacionados às suas características, de modo que, embasados em dados reais e concretos, tenham condições para planejar e prestar assistência individualizada para favorecer a adesão ao tratamento com o controle efetivo da doença [12].

Assim, o presente estudo teve como objetivo avaliar a inter-relação entre o sobrepeso (IMC - índice de massa corpórea), tabagismo, etilismo, sedentarismo, idade, quadros de ansiedade e depressão, bem como fatores socioeconômicos no acometimento de hipertensão em indivíduos atendidos pelo PSF da cidade de Lavras/MG.

\section{Material e métodos}

A pesquisa foi realizada em caráter descritivo, observacional e transversal prospectivo em pacientes atendidos nos PSF da cidade de Lavras, Minas Gerais, Brasil. Na intenção de se avaliar diferentes perfis socioeconômicos, optou-se pela realização de aleatorização dos diferentes unidades do PSF da cidade. Foram incluídas quatro unidades (A, B, C e D) estratificadas dentre as 16 existentes, sendo realizada entrevista através de questionários com moradores residentes em diferentes bairros da cidade (11 no total).

A prevalência média de hipertensos cadastrados dos quatro PSF era de aproximadamente $8 \%$. Por meio de um cálculo prévio sabendo que o pesquisador se dirige intencionalmente a um grupo de elementos (hipertensos) dos quais se deseja saber a opinião, 
optou-se pelo método de amostragem intencional. Após aplicação da fórmula do cálculo do tamanho mínimo da amostra para populações infinitas [13], obteve-se um valor de 113 indivíduos. A entrevista foi realizada com 107 indivíduos e levando-se em consideração a perda amostral de $20 \%$, a amostra foi considerada adequada. O questionário envolvia questões relativas a dados pessoais e hábitos como tabagismo, etilismo, sedentarismo, idade, bem como fatores socioeconômicos e uso de medicamentos. Para fins de pesquisa, os participantes foram apresentados a um questionário de avaliação dos níveis de ansiedade e depressão [14]. Somente foram entrevistados indivíduos acima de 18 anos de idade. A seleção dos entrevistados foi feita de modo aleatório nos próprios PSF's por meio do método da loteria. As entrevistas foram realizadas através de visitas domiciliares. O período de coleta foi de abril a maio de 2009.

A aferição da pressão arterial foi realizada na parte da manhã com esfigmomanômetro manual (Becton-Dickinson) após, pelo menos, dez minutos de descanso, com o entrevistado sentado e com o braço comodamente apoiado ao nível do coração e descoberto. A aferição foi repetida duas vezes para confirmação de valores em todos os indivíduos. O peso e a altura foram avaliados com balança antropométrica e os valores foram obtidos a partir dos prontuários dos indivíduos. As demais perguntas foram respondidas pelo entrevistado e anotadas após leitura e explicação das mesmas.

Todos os participantes forneceram seu consentimento livre e esclarecido por escrito, para que os dados pudessem ser utilizados para fins de pesquisa, segundo a Resolução 196/96 do Ministério da Saúde. O projeto foi aprovado pelo Comitê de Ética em pesquisas com seres humanos do Centro Universitário de Lavras (Unilavras), número do CAAE 0097.0.189.000-09.

Os dados foram analisados por meio de estatística descritiva que consistiu de média, desvio padrão, erro padrão e porcentagens. Foram aplicados testes de regressão logística uni e multivariada para avaliação dos possíveis fatores associados à variável dependente "hipertensão" utilizando-se o Programa SPSS 9.0. A variável desfecho "hipertensão" foi definida a partir do diagnóstico médico do PSF correspondente confirmado por valores pressóricos diastólicos acima de $90 \mathrm{mmHg}$. Após realização do modelo univariado, as variáveis que apresentaram valor de $p<0,30$ foram incluídas no modelo de regressão multivariada.

Resultados

I.

Os dados descritivos relativos aos voluntários avaliados estão demonstrados na tabela

Tabela I - Peso, altura, IMC (índice de massa corpórea) e pressão arterial médios de homens e mulheres voluntários avaliados no PSF no município de Lavras/MG, 2010.

\begin{tabular}{lll}
\hline & Homens & Mulheres \\
\hline Número de indivíduos (N) & 31 & 76 \\
Indice de massa corpórea (IMC) & 27,70 & 26,56 \\
Id ade (anos) & 58,46 & 47,08 \\
Peso (kg) & 77,00 & 68,58 \\
Altura (m) & 1,67 & 1,60 \\
Valores pressóricos sistólicos $(\mathrm{mmHg})$ & 124,00 & 125,45 \\
Valores pressóricos diastólicos $(\mathrm{mmHg})$ & 81,61 & 78,3 \\
\hline
\end{tabular}

Nos resultados do modelo de regressão univariada observou-se que o índice de massa corpórea (IMC) $(p=0,04)$, a idade $(p=0,00)$, o consumo de álcool $(p=0,22)$, a renda familiar acima de um salário mínimo $(p=0,23)$, o número de dependentes abaixo de quatro indivíduos $(p=0,23)$, o uso de medicamentos para hipertensão $(p=0,04)$ e os altos níveis de ansiedade $(p=0,10)$ estiveram positivamente associados à variável desfecho "hipertensão" ao passo que o uso de antidepressivos $(p=0,25)$ e a prática de exercícios físicos $(p=0,15)$ estiveram negativamente associados (Tabela II). 
Tabela II - Análise de regressão univariada para a variável dependente "hipertensão".

\begin{tabular}{|c|c|c|c|c|c|c|}
\hline & & Não & Sim & Total & Valor $p$ & Coeficiente \\
\hline Gênero & $\begin{array}{l}\text { masc } \\
\text { fem }\end{array}$ & $\begin{array}{l}18 \\
50\end{array}$ & $\begin{array}{l}13 \\
26\end{array}$ & $\begin{array}{l}31 \\
76\end{array}$ & 0,45 & $-0,3285$ \\
\hline IMC & $\begin{array}{l}\text { média } \\
\text { DP }\end{array}$ & $\begin{array}{l}26,29 \\
3,95\end{array}$ & $\begin{array}{l}28,24 \\
5,26\end{array}$ & $\begin{array}{l}27,00 \\
4,55\end{array}$ & $0,04^{*}$ & 0,0984 \\
\hline Sobrepeso & $\begin{array}{l}\text { não } \\
\text { simm }\end{array}$ & $\begin{array}{l}26 \\
42\end{array}$ & $\begin{array}{l}12 \\
27\end{array}$ & $\begin{array}{l}38 \\
69\end{array}$ & 0,44 & 0,3314 \\
\hline Id ade (anos) & $\begin{array}{l}\text { média } \\
\text { DP }\end{array}$ & $\begin{array}{l}43,81 \\
15,63\end{array}$ & $\begin{array}{l}55,69 \\
12,77\end{array}$ & $\begin{array}{l}48,14 \\
15,68\end{array}$ & $0,00^{\circ}$ & 0,0537 \\
\hline Fumo & $\begin{array}{l}\text { não } \\
\text { simm }\end{array}$ & $\begin{array}{l}63 \\
5\end{array}$ & $\begin{array}{l}35 \\
4\end{array}$ & $\begin{array}{l}98 \\
9\end{array}$ & 0,60 & 0,3646 \\
\hline Álcool & กão & $\begin{array}{l}64 \\
4\end{array}$ & $\begin{array}{l}34 \\
5\end{array}$ & 98 & $0,22^{\circ}$ & 0,8557 \\
\hline Exercícios & $\begin{array}{l}\text { não } \\
\text { sim }\end{array}$ & $\begin{array}{l}43 \\
25\end{array}$ & $\begin{array}{l}30 \\
9\end{array}$ & $\begin{array}{l}73 \\
34\end{array}$ & $0,15^{\circ}$ & $-0,6616$ \\
\hline Renda (> 1 salário) & $\begin{array}{l}\text { não } \\
\text { sim }\end{array}$ & $\begin{array}{l}27 \\
41\end{array}$ & $\begin{array}{l}11 \\
28\end{array}$ & $\begin{array}{l}38 \\
69\end{array}$ & $0,23^{\circ}$ & 0,5166 \\
\hline Número de dependentes ( $<4$ ) & $\begin{array}{l}\text { não } \\
\text { simm }\end{array}$ & $\begin{array}{l}29 \\
39\end{array}$ & $\begin{array}{l}12 \\
27\end{array}$ & $\begin{array}{l}41 \\
66\end{array}$ & $0,23^{-}$ & 0,5147 \\
\hline Medicamento para hiperten são & $\begin{array}{l}\text { não } \\
\text { sim }\end{array}$ & $\begin{array}{l}42 \\
26\end{array}$ & $\begin{array}{l}16 \\
23\end{array}$ & $\begin{array}{l}58 \\
49\end{array}$ & $0,04^{*}$ & 0,8425 \\
\hline Parente com hipertensão & $\begin{array}{l}\text { não } \\
\text { sim }\end{array}$ & $\begin{array}{l}16 \\
51\end{array}$ & $\begin{array}{l}10 \\
29\end{array}$ & $\begin{array}{l}26 \\
80\end{array}$ & 0,48 & $-0,2205$ \\
\hline Diabetes & $\begin{array}{l}\text { não } \\
\text { sim }\end{array}$ & $\begin{array}{l}62 \\
6\end{array}$ & $\begin{array}{l}33 \\
6\end{array}$ & $\begin{array}{l}95 \\
12\end{array}$ & 0,31 & 0,6306 \\
\hline Uso de antidepressivo & $\begin{array}{l}\text { não } \\
\text { sim }\end{array}$ & $\begin{array}{l}51 \\
17\end{array}$ & $\begin{array}{l}33 \\
6\end{array}$ & $\begin{array}{l}84 \\
23\end{array}$ & 0,25 & $-0,6061$ \\
\hline An siedade & $\begin{array}{l}\text { não } \\
\text { sim }\end{array}$ & $\begin{array}{l}55 \\
13\end{array}$ & $\begin{array}{l}26 \\
13\end{array}$ & $\begin{array}{l}81 \\
26\end{array}$ & $0,10^{-}$ & 0,7492 \\
\hline Depressão & $\begin{array}{l}\text { não } \\
\text { sim }\end{array}$ & $\begin{array}{l}52 \\
16 \\
68\end{array}$ & $\begin{array}{l}32 \\
7 \\
39\end{array}$ & $\begin{array}{l}84 \\
23 \\
107\end{array}$ & 0,50 & $-0,3412$ \\
\hline
\end{tabular}

${ }^{*} p<0,30$ (limite para variáveis independentes serem incluídas no modelo multivariado).

Em um segundo momento, foi aplicado modelo de regressão logística multivariada (stepwise forward procedure) de forma a identificar quais destas variáveis independentes ainda apresentavam influência sobre o desfecho "presença de hipertensão" após o controle da interação entre as diversas variáveis independentes investigadas (Tabela III).

Tabela III - Análise de regressão logística multivariada (stepwise forward procedure) para a variável dependente hipertensão.

\begin{tabular}{|c|c|c|c|c|}
\hline & $\begin{array}{l}\text { Odds Ratio (Confidence } \\
\text { Interval) }\end{array}$ & valor de $p$ & Coeficiente & $\mathrm{DP}$ \\
\hline IMC & $\begin{array}{l}\text { Referência } \\
1.19(1.04 \text { a } 1,36)\end{array}$ & $0,009^{\circ}$ & 0,175 & 0,067 \\
\hline Id ade & $\begin{array}{l}\text { Referência } \\
1,11(1,06 \text { a } 1,17)\end{array}$ & $<0,0001^{\circ}$ & 0,104 & 0,026 \\
\hline Álcool & $\begin{array}{l}\text { Referência } \\
15.19(2.13 \text { a 108.45) }\end{array}$ & $0,006^{\circ}$ & 2,721 & 1,002 \\
\hline Antidepressivos & $\begin{array}{l}\text { Referência } \\
0,15(0,03 \text { a } 0,72)\end{array}$ & $0,016^{*}$ & $-1,846$ & 0,771 \\
\hline An siedade & $\begin{array}{l}\text { Referência } \\
4,93(1,37 \text { a } 17,75)\end{array}$ & $0,014^{\circ}$ & 1,596 & 0,653 \\
\hline
\end{tabular}

\section{Discussão}

Observou-se que as variáveis "índice de massa corpórea" (IMC) e "idade" aumentaram ligeiramente a chance de o indivíduo apresentar hipertensão (Odds ratio: 1,19 e 1,11 respectivamente). Isto significa que um indivíduo com IMC $>25$ (sobrepeso e obeso) e mais velho apresentou maior risco de apresentar hipertensão. 
Entretanto, níveis aumentados de ansiedade e o consumo de álcool acima de três vezes por semana aumentaram, em aproximadamente, 5 (Odds ratio: 4,93) e 15 (Odds ratio: $15,19)$ vezes, respectivamente, as chances de o indivíduo apresentar hipertensão. Isso indica que o consumo de álcool e níveis aumentados de ansiedade intensificam as chances de o indivíduo ser hipertenso. Por outro lado, o uso de medicamentos antidepressivos diminuiu ligeiramente (Odds ratio: 0,15$)$ a chance de acometimento por hipertensão, sugerindo que 0 controle do estado emocional exerce efeito protetor contra a hipertensão.

Todavia, hábitos alimentares são importantes para a reversão do desequilíbrio que ocorre na HAS. Isto pode ser feito através do controle da ingestão de sódio e calorias, reduzindo e posteriormente mantendo o peso corporal [17]. Os achados elevados de sobrepeso e obesidade reforçam a necessidade de implantação de medidas objetivas para 0 seu combate, com vistas à redução da morbidade e da mortalidade por doença cardiovascular.

A prática de exercícios físicos no presente estudo esteve negativamente associada à hipertensão, de forma que, quanto menos atividade física o indivíduo pratica, maior o risco de alteração cardiovascular. Pessoas fisicamente ativas possuem menor incidência de hipertensão arterial [18]. A prática de atividade física regular demonstra a opção por um estilo de vida mais ativo, relacionado ao comportamento humano voluntário no qual se integram componentes e determinantes de ordem biológica e psico-sócio-cultural. Porém, indivíduos hipertensos sempre devem procurar atendimento médico antes de iniciar qualquer atividade física.

Níveis aumentados de ansiedade e o consumo de álcool acima de três vezes por semana aumentaram, em aproximadamente, 5 (Odds ratio: 4,93) e 15 (Odds ratio: 15,19) vezes, respectivamente, as chances do indivíduo apresentar hipertensão. Isso indica que o consumo de álcool e níveis aumentados de ansiedade intensificam as chances de o indivíduo ser hipertenso. Por outro lado, o uso de medicamentos antidepressivos diminuiu ligeiramente (Odds ratio: 0,15 ) a chance de acometimento por hipertensão, sugerindo que o controle do estado emocional exerce efeito protetor contra a hipertensão.

O consumo de álcool com alta frequência contribuiu significativamente para o aumento das chances do indivíduo apresentar hipertensão arterial. Estima-se que $10 \%$ dos pacientes hipertensos tenham exacerbação dos níveis pressóricos quando fazem uso de álcool. $\mathrm{O}$ mecanismo exato que leva à hipertensão não é totalmente conhecido, entretanto, são descritos fatores centrais e periféricos, como estimulação do sistema nervoso simpático e do sistema renina-angiotensina-aldosterona, além de uma disfunção arterial [19].

No presente estudo, o hábito de fumar não apresentou associação com o desfecho hipertensão. Entretanto, este resultado negativo deve ser avaliado com cautela, uma vez que somente nove pacientes eram fumantes. Esta característica foi considerada inerente da amostra avaliada, de forma que medidas antitabagistas prévias executadas pelos profissionais atuantes nos referidos PSFs resultaram na interceptação deste fator nas populações atendidas. Desta forma, ressalta-se que o tabagismo está intimamente associado à hipertensão e que medidas preventivas quanto a este hábito podem favorecer a diminuição dos índices de HAS [20].

O uso de medicamentos antidepressivos diminuiu ligeiramente a chance de acometimento por hipertensão nos voluntários avaliados, o que sugere que um controle do estado emocional exerce efeito protetor contra a hipertensão. Em contrapartida, indivíduos com altos níveis de ansiedade apresentaram maior propensão a desenvolverem hipertensão. Essa situação evidencia a importância do estudo das causas de ordem psicológica, capazes de repercutir sobre 0 organismo humano. Hipertensos complicados apresentam mais características estruturais e psicossociais desfavoráveis, mais atitudes negativas frente ao tratamento e desconhecem a doença [12], reforçando a ideia de que o aspecto biopsicossocial é de importância na avaliação de pacientes hipertensos pelos profissionais de saúde de PSF. Adicionalmente, características da personalidade como sentimento de bem-estar, socialização e capacidade de se sentir igual a seus pares podem também se associar com a adesão ao tratamento, contribuindo para o controle da doença [21].

Os fatores socioeconômicos como renda e número de residentes na casa também alcançaram valores suficientes para entrar na análise multivariada. Porém, estes fatores foram eliminados, provavelmente em razão da sobreposição com a variável IMC. No que se refere à condição socioeconômica e a sua relação com a hipertensão, estes conduzem a sua correlação com um estilo de vida não saudável. Adicionalmente, indivíduos com melhor condição econômica apresentam maior acesso a alimentos industrializados e com grandes quantidades de calorias. Em relação às condições socioeconômicas e o comportamento 
nutricional, foi observado que ambos influenciam na ocorrência de HAS. Os fatores socioeconômicos (incluindo oportunidades educacionais e de trabalho) podem afetar os índices e prevalência de HAS [15].

\section{Conclusão}

Conclui-se que hábitos de vida sedentários, associados ao tabagismo, ansiedade e consumo de álcool, correlacionam-se positivamente com a prevalência de hipertensão na amostra avaliada. Desta forma, métodos de controle do peso corporal, da ansiedade e a diminuição do consumo de bebidas alcoólicas devem ser enfatizados na atuação de enfermeiros que atuam em PSF.

1. Duarte MTC, Cyrino AP, Cerqueira ATAR, Nemes MIB, lyda M. Reasons for medical folow-up dropout among patients with arterial hypertension: the patient's perspective. Ciênc Saúde Coletiva 2010;15:2603-10.

2. Mion Junior D, Kohlmann Junior O, Machado CA, Amodeo C, Gomes MAM, Praxedes JN et al. Sociedade Brasileira de Cardiologia. Sociedade Brasileira de Hipertensão/ Sociedade Brasileira de Nefrologia. V Diretrizes Brasileiras de Hipertensão Arterial. Arq Bras Cardiol 2007;89:24-79.

3. Banegas JR, Rodríguez-Artalejo F, Ruilope LM, Graciani A, Luque M, de la Cruz-Troca $\mathrm{JJ}$ et al. Hypertension magnitude and management in the elderly population of Spain. $J$ Hypertens 2002;20:2157-64.

4. Gaudemaris R, Lang T, Chatellier G, Larabi L, Lauwers-Cancès V, Maître A et al. Socioeconomic inequalities in hypertension: Prevalence and care. Hypertension 2002;39:1119-25.

5. Rodrigues Barbosa A, Ferreti Borgatto A. Arterial hypertension in the elderly of Bridgetown, Barbados: prevalence and associated factors. J Aging Health 2010;22:61130.

6. Kearney PM, Whelton M, Reynolds K, Muntner P, Whelton PK, He J. Global burden of hypertension: analysis of worldwide data. Lancet 2005;365:217-23.

7. Lawes CM, Vander Hoorn S, Rodgers A; International Society of Hypertension. Global burden of blood-pressure-related disease, 2001. Lancet 2008;371:1513-8.

8. Inamo J, Lang T, Atallah A, Inamo A, Larabi L, Chatellier G et al. Prevalence and therapeutic control of hypertension in French Caribbean regions. J Hypertens 2005;23:1341-6.

9. Rossi R, Nuzzo A, Modena MG. The management of pre-hypertension and metabolic syndrome. Minerva Cardioangiol 2009;57:723-31.

10. Monteiro LZ, Fiani CR, Freitas MC, Zanetti ML, Foss MC. Decrease in blood pressure, body mass index and glycemia after aerobic training in elderly women with type 2 diabetes. Arq Bras Cardiol 2010;95:563-70.

11. Padwal RS, Hemmelgarn BR, Khan NA, Grover S, McKay DW, Wilson T et al. The 2009 Canadian Hypertension Education Program recommendations for the management of hypertension: Part 1- Blood pressure measurement, diagnosis and assessment of risk. Can J Cardiol 2009;25:279-86.

12. Pierin AM, Jesus ED, Augusto MA, Gusmão J, Ortega K, Mion Junior D. Biopsychosocial variables and attitudes towards treatment influence complicated hypertension. Arq Bras Cardiol 2010;95:648-54.

13. Cochran WG. Sampling Techniques. 3rd ed. New York: John Wiley; 1977.

14. Botega NJ, Bio MR, Zomignani MA, Garcia C Jr, Pereira WA. Mood disorders among inpatients in ambulatory and validation of the anxiety and depression scale HAD. Rev Saúde Pública 1995;29:355-63.

15. Nascente FM, Jardim PC, Peixoto MD, Monego ET, Moreira HG, Vitorino PV et al. Arterial hypertension and its correlation with some risk factors in a small Brazilian town. Arq Bras Cardiol 2010;95(4):502-8. 
16. Goodpaster BH, Delany JP, Otto AD, Kuller L, Vockley J, South-Paul JE et al. Effects of diet and physical activity interventions on weight loss and cardiometabolic risk factors in severely obese adults: a randomized trial. JAMA 2010;304:1795-802.

17. Weinberger MH. More novel effects of diet on blood pressure and lipids. JAMA 2005;294:2497-8.

18. Carnethon MR, Evans NS, Church TS, Lewis CE, Schreiner PJ, Jacobs DR Jr et al. Joint associations of physical activity and aerobic fitness on the development of incident hypertension: coronary artery risk development in young adults. Hypertension 2010;56:49-55.

19. Rahmouni K, Correia ML, Haynes WG, Mark AL. Obesity-associated hypertension: new insights into mechanisms. Hypertension 2005;45:9-14.

20. Carandang R, Seshadri S, Beiser A, Kelly-Hayes M, Kase CS, Kannel WB, et al. Trends in incidence, lifetime risk, severity, and 30-day mortality of stroke over the past 50 years. JAMA 2006;296:2939-46.

21. Esler M, Schwarz R. Management of hypertension complicated by psychiatric comorbidity. J Clin Hypertens 2007;9:714-5. 